PIERS_2019 Rome

\title{
Electromagnetic Analysis and Performance Comparison of Fully 3D-Printed Antennas
}

\author{
R. Colella ${ }^{1}$, F. Chietera ${ }^{1}$, A. Casula ${ }^{2}$, A. Michel ${ }^{3}$, G. Montisci ${ }^{2}$, G. Gatto ${ }^{2}$, P. Nepa ${ }^{3}$, H. Rogier ${ }^{4}$, L. Catarinucci ${ }^{1}$ \\ ${ }^{1}$ Innovation Engineering Department, University of Salento - Lecce, Italy \\ ${ }^{2}$ Department of Electrical and Electronic Engineering, University of Cagliari, Cagliari, Italy \\ ${ }^{3}$ Department of Information Engineering, University of Pisa, Pisa, Italy \\ ${ }^{4}$ Internet Technology and Data Science Lab, Ghent University, Gent, Belgium
}

\begin{abstract}
In this work, the possibility of directly prototyping antennas by exploiting additive manufacturing 3D-printing technology is investigated. In particular, the availability of printable filaments with interesting conductive properties allows for the printing even of the conductive elements. Three samples of a $2.45 \mathrm{GHz}$ microstrip patch antenna have been 3D-printed by using different approaches and materials, and then performance evaluated and compared. In particular, the same dielectric substrate printed in polylactic acid (PLA) has been adopted in all cases, whilst copper tape and two different conductive filaments have been used to realize the conductive parts of the three antenna samples, respectively. The comparative analysis definitely demonstrates that 3D-printing technology can be exploited to design efficient low-cost antennas.
\end{abstract}

\section{INTRODUCTION}

In the recent years, additive manufacturing has been receiving an increasing attention to create low-cost structures with custom size and shapes. In particular, 3-D printing represents a key technology to enable the design of new and challenging antennas more efficient and compact. For example, in [1], a 3D-printed air substrate has been fabricated for high-gain patch antenna. In [2], a circularly polarised (CP) patch antenna has been comprised by 3D-printed substrate and inkjet-printed metal. Also, in [3] a microstrip patch antenna with circular polarisation is presented by using an intrinsically asymmetric 3D-printed substrate. Some of the authors also proposed a 3D printed antenna for UHF RFID handheld readers [4] and compact 3D-printed wearable antenna feeding a UHF RFID sensor tag designed to passively sense and retrieve the human body temperature [5]. Nevertheless, the 3D printing can be reliably used also to design RF components such as T-resonators or metamaterial-inspired phase shifter [5-6].

It is worth noting that the fully dielectric characterization of the 3-D printed substrate is fundamental for designing RF devices or antennas. For this reason, studies aimed at characterizing new 3D-printed materials mainly in terms of dielectric properties have been published in the last years [7-8]. In [7] authors measure a wide variety of 3D-printed materials such as ABS, PTFE, and many others over a broad spectrum of frequencies from $1 \mathrm{MHz}$ to $10 \mathrm{GHz}$ using a variety of well-established measurement methods. In [8] a waveguide-based method has been used for the measurement of dielectric constants of three types of polylactic acids (PLAs). Moreover, in order to discuss the dependence of the dielectric properties of 3D-printed PLA structures when varying infill percentage and infill shape, a commercial split-post-dielectric resonator and a T-resonator have been used in [9] and [10], respectively.

On the other hand, different metal printing methods have been developed to create a metallization on the 3D 
printed substrate, such as SLA (stereolithography), fused powders, polyjet, fused layering of sheets, ink jetted resin, FDM (fuse deposition modeling), or SLS (selective laser sintering). A comprehensive study of how different plastics materials, metallization processes, and thickness of the metal impact the performance of an antenna can be found in [11] for the particular case of an horn antenna.

In this paper, a comparative numerical and experimental analysis of three different 3D-printed $2.45 \mathrm{GHz}$ microstrip patch antennas is presented. Specifically, all the three antenna samples are fabricated by using the same dielectric PLA substrate. The conductive parts of the first sample have been realized by using a properly shaped adhesive copper tape, so as to consider this antenna as the reference sample. Conversely, two different 3D-printable conductive filaments have been adopted in the other two cases, thus obtaining two samples of fully-3D-printed antennas useful for the comparative analysis. The paper is organized as follows: in Section 2, the design and the prototyping process of the three antennas are analyzed. In Section 3, measured results in terms of reflection coefficient, realized gain and radiation pattern are presented and discussed. In Section 4, conclusions are drawn.

\section{3D-PRINTING ANTENNA DESIGN AND PROTOTYPING}

As stated, the main goal of this work is to realize and characterize fully 3D-printed antennas. For this reason, three samples of an edge-fed microstrip patch antenna working at around $2.45 \mathrm{GHz}$ have been designed and prototyped using different techniques but sharing the same design, which is shown in Fig. 1.

More specifically, Sample 1 has been realized in a hybrid way. On the one hand, the antenna substrate has been produced through additive manufacturing 3D printing technique by using the PLA (Polylactic Acid) as printing

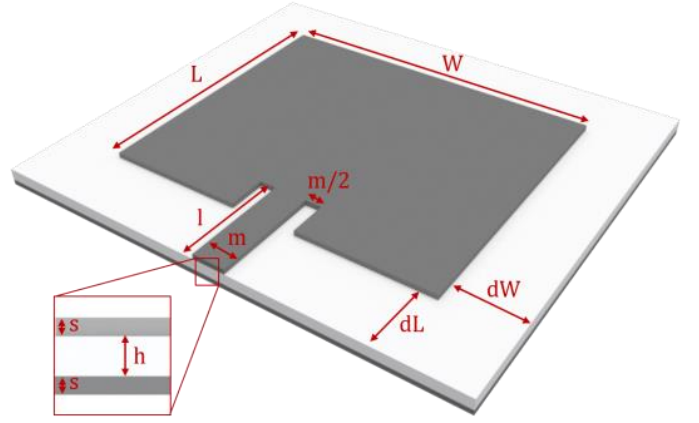

Figure 1. 3D model of the generic patch antenna with its main dimensions material. On the other hand, all the conductive parts, consisting in ground-plane the radiating element, have been realized by properly shaping an adhesive copper tape to be applied on the 3D-printed substrate in a separate process.

Conversely, Sample 2 has been fully realized with a 3D-printing process. In particular, while the PLA has been maintained to print the antenna substrate, all the conductive parts have been 3D-printed with a special conductive filament composed of both PLA and copper particles. This filament is called Electrifi and it is developed by the Duke University to empower the fully 3D-printed electronics. At the authors' knowledge the filament has been already characterized for low frequency applications [12], and used to realize an example of 3D-prineted microstrip waveguide [13]. However, no examples of applications on antenna design have been published so far. Similarly to the Sample 2, the antenna Sample 3 has been designed to fulfill a fully 3D printing process, even though an alternative conductive filament has been used to design the radiating parts. Specifically, a PLA-based material enhanced with graphene particles called BlackMagic [14].

As better clarified next paragraphs, the choice of designing three antennas with similar features but different composition helps in characterizing the novel 3D-printable conductive filaments. Indeed, the electromagnetic performance of both fully-3D antennas (Sample 2 and Sample 3) may be straightforwardly compared with the more predictable ones obtainable with the copper-based antenna (Sample 1). 


\section{A. Design and simulation with CST Microwave Studio}

Design and simulations of the three antenna samples have been performed using CST Microwave Studio Suite [15]. The well-known analytical formulation of the patch antenna has been considered to preliminarily determine the dimensions of the three antennas samples used as starting point of the simulation. Consequently, each sample has been properly refined in the simulation environment according the specific fabrication requirements.

More specifically, an homogenous material with permittivity $\varepsilon_{\mathrm{r}}=2.59$ and $\tan \delta=0.0158$ has been considered to simulate the PLA according to the experimental results reported in [16]. In order to make easier the comparative analysis, the PLA material has been used to model all the three antenna substrates. However, substantial differences are in the definition of the conductive materials, mainly in terms of conductivity and thickness.

For instance, the metallic parts of the Sample 1 have been designed in CST MW Studio by using copper annealed $\left(\sigma=5.7 \cdot 10^{7} \mathrm{~S} / \mathrm{m}\right)$ as material to define a laminate of thickness equal to $35 \mu \mathrm{m}$ simulating the copper tape applied on the PLA substrate.

Once defined, the Sample 1 antenna has been optimized and a resonance at $2.45 \mathrm{GHz}$ has been observed. Contextually, the length of the 50-ohm microstrip line feeding the antenna (and then the antenna inset) has been tuned to guarantee the impedance matching with a 50-ohm port. With reference to Fig. 1, the values of the antenna parameters for this configuration are reported in the first column of Table 1.

Differently form the Sample 1, the antenna Sample 2 has been simulated by using a lossy metal template material to describe the Electrifi properties. In particular, a conductivity $\sigma=1.66 \cdot 10^{4} \mathrm{~S} / \mathrm{m}$ has been considered, according to linear resistivity of about $0.006 \Omega \cdot \mathrm{cm}$ provided by the manufacturer. Also in this case the structure has been properly optimized to match an input impedance of $50 \Omega$ and a resonance at $2.335 \mathrm{GHz}$ has been obtained with the antenna parameters reported in the second column of Table 1.

Finally, the BlackMagic material used to define the

\begin{tabular}{r|ccc} 
& $\begin{array}{c}\text { Sample 1 } \\
\text { Copper }\end{array}$ & $\begin{array}{c}\text { Sample 2 } \\
\text { Electrifi }\end{array}$ & $\begin{array}{c}\text { Sample 3 } \\
\text { BlackMagic }\end{array}$ \\
\hline $\mathbf{L}$ & 37.4 & 37.2 & 37.2 \\
$\mathbf{W}$ & 475 & 44.5 & 44.5 \\
$\mathbf{d L}$ & $12 \cdot \mathrm{h}$ & $12 \cdot \mathrm{h}$ & $12 \cdot \mathrm{h}$ \\
$\mathbf{d W}$ & $12 \cdot \mathrm{h}$ & $12 \cdot \mathrm{h}$ & $12 \cdot \mathrm{h}$ \\
$\mathbf{h}$ & 1.5 & 1.5 & 1.5 \\
$\mathbf{S}$ & 0.035 & 0.7 & 0.7 \\
$\mathbf{I}$ & 11 & 5 & 5 \\
$\mathbf{m}$ & 4.3 & 4.3 & 4.3
\end{tabular}

Table 1. Sample 1,2 and 3 sizes in [mm] with respect to the nomenclature shown in Figure 1. conductive parts of the antenna Sample 3 has been modeled with a $\sigma=1.66 \cdot 10^{2} \mathrm{~S} / \mathrm{m}$, according to the linear resistivity value of $0.6 \Omega \cdot \mathrm{cm}$, provided by the manufacturer. This extremely low conductivity value (which is two orders of magnitude lower than the Electrifi one) seems to compromise the radiation properties of this antennas. Indeed, no clear resonance frequencies are highlighted by the simulation. However, the antenna having the size defined by the dimensional parameters reported in third column of Table 1 has been considered for realization thus experimentally confirming/un-confirming this simulated outcome.

It is worth highlighting that, differently from Sample 1, the other two antenna samples exhibit a conductive parts thickness of $700 \mu \mathrm{m}$ instead of $35 \mu \mathrm{m}$. This considerable difference is due to practical needs. In fact, although a neglectable impact has been observed on the antenna performance when varying this thickness, a considerable advantage in terms of physical robustness of structure is expected once the antenna is $3 \mathrm{D}$-printed. 


\section{B. The prototyping process}

The single-extruder 3D printer shown in Fig. 2 has been used to realize the antenna prototypes. The diameter of the extruder nozzle used for the printing process is $0.5 \mathrm{~mm}$ while the layer height is variable but always under $0.2 \mathrm{~mm}$. It is worth observing how these values are perfectly compatible with the antennas working frequency. In fact, the wavelength at $2.4 \mathrm{GHz}$ is largely greater than the highest possible imperfection due to the printing

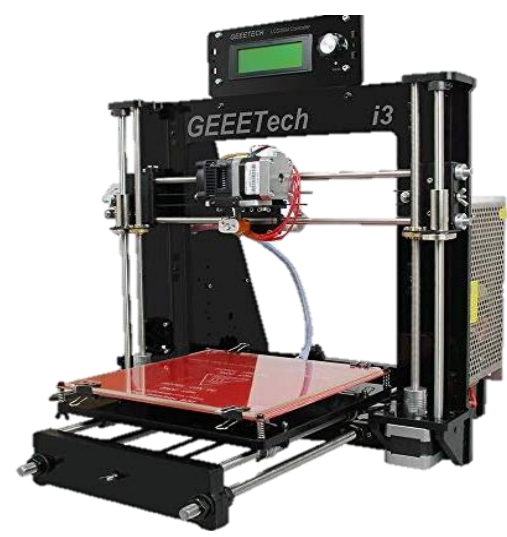

Figure 2. FDM 3D printer model Geeetech Prusa I3 Pro B. process, and this allows to consider the printed parts as homogeneous in the selected range of frequencies.

As stated, Sample 1 has been produced with a hybrid technique, and the only 3D-printed part has been the PLA substrate whilst its metallic parts have been shaped onto an adhesive copper tape through a cutting plotter Graphtec CE6000 Series and then attached on the substrate.

Instead, Sample 2 and Sample 3 have been fully 3D printed. The three elements of each antenna (background, substrate, and patch) have been sequentially printed one above the other, pausing the printing when necessary to allow the change of the filament and the cleaning of the extruder.

In Table 2, the main printing settings of the three filaments used in this work are presented.

Another important parameter to be taken into account is the retraction coefficient of the plastic materials extruded by the printer. In fact, every material, even the most stable one, exhibits some kinds of retraction during the printing process. This is a tolerable imperfection in most of the cases, especially if the retraction coefficient is sufficiently low. However, even changes in the order of the millimeter fraction can hugely impact on hi-frequency applications and need to be considered during the design procedure.

\begin{tabular}{r|ccc} 
& PLA & Electrifi & BlackMagic \\
\hline Extr. Temp. $\left[{ }^{\circ} \mathbf{C}\right]$ & 205 & 145 & 195 \\
Bed Temp. $\left[{ }^{\circ} \mathbf{C}\right]$ & 35 & 35 & 35 \\
Speed $[\mathrm{mm} / \mathbf{m i n}]$ & 2600 & 600 & 2000 \\
Layer height $[\mathbf{m m}]$ & 0.2 & 0.15 & 0.15
\end{tabular}

Table 2. Printing settings of the different printed materials.

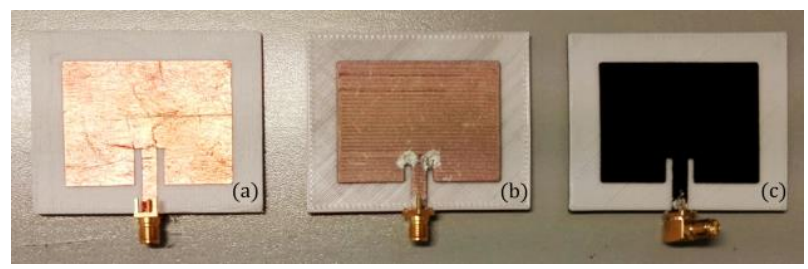

Figure 3. The three realized antenna prototypes: Sample 1 (a), Sample 2 (b) and Sample 3 (c).
In particular, for the Sample 2 antenna, a retraction of the Electrifi led to a reduction of the antenna dimensions of about $0.15 \mathrm{~mm}$ on both $\mathrm{x}$ and $\mathrm{y}$ axis, determining a slightly increment of the resonant frequency compared with the simulation results.

For the three antennas, standard SMA connectors have been used. However, while on Sample 1 a soldering procedure has been performed, the connection with conductive parts in Sample 2 and Sample 3, has been guaranteed through a conductive glue since the plastic matrix of the conductive materials is not compatible with high-temperature soldering. The conductive glue used for that purpose has been the 8331 silver conductive epoxy adhesive, from MGChemicals ${ }^{\circledR}$ [17]. This glue dries in 24 hours at room temperature and exhibits a linear resistivity of $0.007 \Omega \cdot \mathrm{cm}$. Moreover, the glue is useful to solve the problems related to high 
values of contact resistance, which are common using these plastic-based conductive materials. In Fig. 3 a picture of the three realized samples is shown.

\section{RESULTS AND DISCUSSION}

The three designed antennas have been fully characterized in an anechoic chamber. In Fig 4 the reflection coefficients simulated using CST Microwave Studio are compared with the results measured by a commercial VNA.

The measured frequency response for the standard microstrip patch implemented with copper tape, the sample 1, is very close to the simulated one. The resonant frequency is $2.44 \mathrm{GHz}$, and the $-10 \mathrm{~dB}$ bandwidth is $60 \mathrm{MHz}$ for the simulated patch and $40 \mathrm{MHz}$ for the measured prototype.

On the other hand, the sample 3, i.e. the patch implemented using the graphene filament, does not radiate, and its measured frequency response monotonically decreases, showing no resonating behavior, with the S11 module equal to $-15 \mathrm{~dB}$ at $2 \mathrm{Ghz}$ and to $-24 \mathrm{~dB}$ at $3 \mathrm{GHz}$. This antenna dissipates almost all the input power, and this is due both to the very low conductivity of the graphene filament (the estimated conductivity is only $\sigma=160 \mathrm{~S} / \mathrm{m}$ ), and to the mixture between the graphene and the insulating matrix of PLA used to form the graphene-based filament, which could cause the presence of parasitic capacitances on the deposed metallic traces.

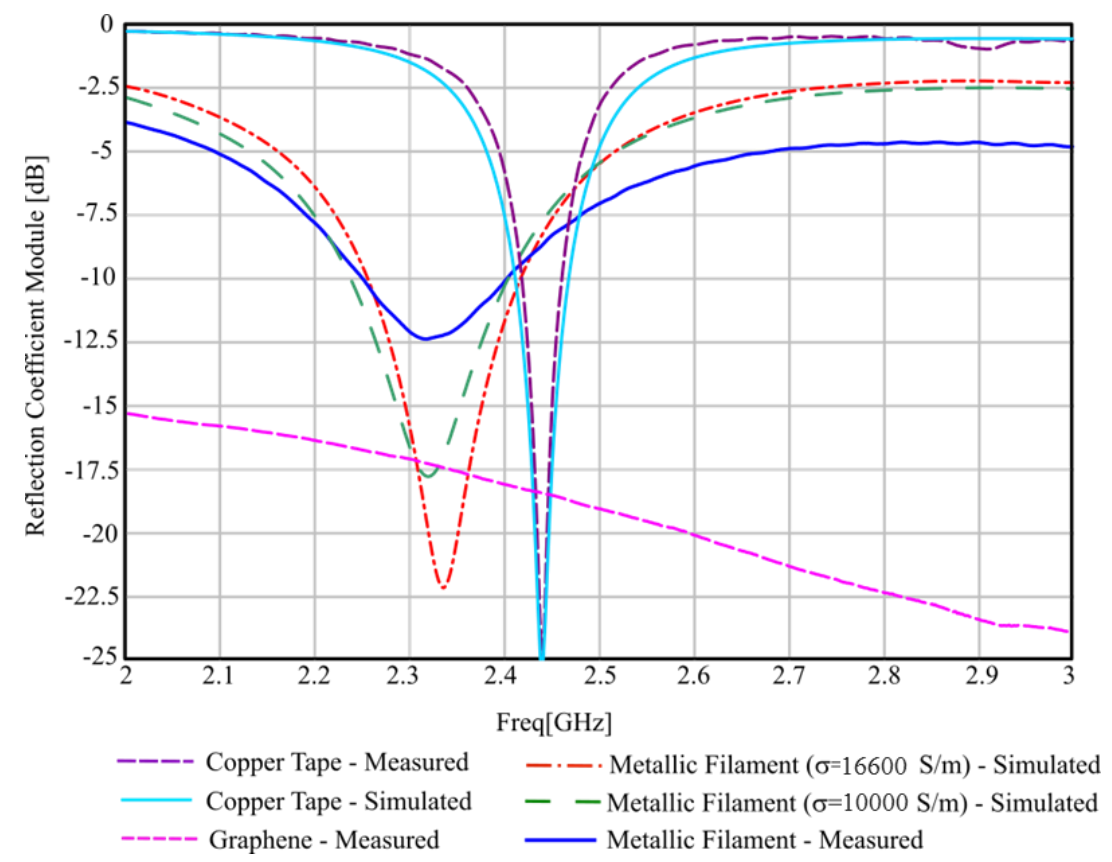

Figure 4. Frequency responses of the designed microstrip patch antennas.

The sample 1, i.e. the patch fully $3 \mathrm{D}$ printed using the Electrifi metallic filament exhibits a measured resonant frequency equal to $2.318 \mathrm{GHz}$, with a $-10 \mathrm{~dB}$ bandwidth of $150 \mathrm{MHz}$. The simulated structure, using the datasheet conductivity of the filament equal to $\sigma=1.66 \cdot 10^{4} \mathrm{~S} / \mathrm{m}$, presents a frequency shift, resonating at 2.335 $\mathrm{GHz}$, with a $-10 \mathrm{~dB}$ bandwidth of $160 \mathrm{MHz}$, and a peak at the resonance equal to $-22 \mathrm{~dB}$. This behavior is a bit different from the measured prototype, and this can be due to the effective value of the filament conductivity at the operating frequency of the patch antenna. In fact, the value of $\sigma=1.66 \cdot 10^{4} \mathrm{~S} / \mathrm{m}$ given by the datasheet is a static value, i.e. for null frequency. Therefore a lower value of $\sigma$ at $2.4 \mathrm{GHz}$ is expected. In Fig. 4 the simulated response of the filament patch is reported when a $30 \%$-decreased value of $\sigma=10^{4} \mathrm{~S} / \mathrm{m}$ is considered. It can be seen that the frequency response is in this case very close to the measured one, with a resonant frequency of 
$2.319 \mathrm{GHz}$, and a $-10 \mathrm{~dB}$ bandwidth equal to $166 \mathrm{MHz}$. The peak at resonance, equal to $-17.5 \mathrm{~dB}$, is still lower with respect to the measured one (which is $-12.5 \mathrm{~dB}$ ), but it can be due to the mixture between the metal and the PLA to form the metallic filament (which is not pure metal), which could cause the presence of parasitic capacitances on the deposed metallic traces (and therefore producing a different value of $S_{11}$ ) in the realized prototype.

In Fig. 5 the radiation pattern of the designed antennas with copper tape and metallic filament are reported in the horizontal plane. For the copper patch the radiation pattern is very close to a standard microstrip patch antenna, with a front-to-back ratio of $7 \mathrm{~dB}$, a $-3 \mathrm{~dB}$ beamwidth around $70^{\circ}$, and a gain of around $5.5 \mathrm{~dB}$. The patch realized with the metallic filament, instead, has slightly broader radiation pattern, with a similar front-to-back ratio around $7 \mathrm{~dB}$. This behavior could be due to a different current distribution on the patch with respect to the copper one, either because a preferential path for the electrical current can exist (which follows the direction of the deposed filament), either because the metallic filament is not pure metal, but it is mixed with insulating materials causing the presence of parasitic capacitances on the patch surface.

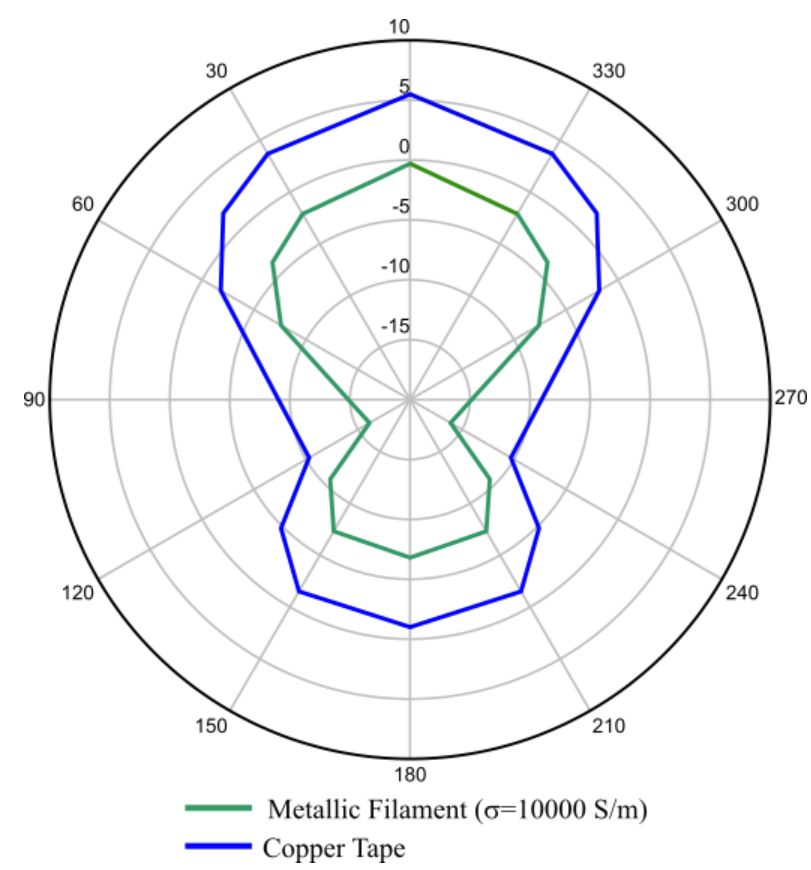

Figure 5. Measured realized Gain in the horizontal plane of the designed microstrip patch antennas.

\section{CONCLUSIONS}

Additive manufacturing 3D printing is a relatively new and rapidly growing technology, with several advantages in many different fields, including electromagnetics. In this work, this technique has been applied for prototyping fully-3D-Printed microstrip patch antennas, with the main goal to investigate the appropriateness of some printable filaments with conductive properties to be used for the direct printing of radiating elements of antennas. Three patch antennas have been designed, realized and tested. The conductive elements of the first of them, used as reference, have been realized by using a properly shaped adhesive copper tape. On the contrary, Electrifi and BlackMagic filaments have been used for the other two, respectively. The efficiency of the Electrifi-based fully-3D-printed prototype resulted satisfactory, although it is a couple of dB lower than the copper-based antenna. The obtained results clearly demonstrates that additive manufacturing 3D-printing technology can be effectively used to implement fully printed antennas including its conductive parts. 
PIERS_2019 Rome

\section{REFERENCES}

1. Ghazali, M.I.M., Karuppuswami, S., Kaur, A., et al.: '3-D printed air substrates for the design and fabrication of RF components', Trans. Compon. Packag. Manuf. Technol., 2017, 7, (6), pp. 982-989,

2. Jun, S., Sanz-Izquierdo, B., Heirons, J., et al.: 'Circular polarised antenna fabricated with low-cost 3D and inkjet printing equipment', Electron. Lett., 2017, 53, (6), pp. 370-371

3. S. Jun et al., "Circular polarised antenna fabricated with low-cost 3D and inkjet printing equipment," in Electronics Letters, vol. 53, no. 6, pp. 370-371, 163 2017. doi: 10.1049/el.2016.4605

4. R. Colella, A. Michel and L. Catarinucci, "Compact 3-D-Printed Circularly Polarized Antenna for Handheld UHF RFID Readers," in IEEE Antennas and Wireless Propagation Letters, vol. 17, no. 11, pp. 2021-2025, Nov. 2018. doi: 10.1109/LAWP.2018.2860253

5. R. Colella and L. Catarinucci, "Wearable UHF RFID Sensor-Tag Based on Customized 3D-Printed Antenna Substrates," in IEEE Sensors Journal, vol. 18, no. 21, pp. 8789-8795, 1 Nov.1, 2018. doi: 10.1109/JSEN.2018.2867597

6. M. I. M. Ghazali, S. Karuppuswami, S. Mondal, A. Kaur and P. Chahal, "Embedded Actives Using Additive Manufacturing For High Density RF Circuits and Systems," in IEEE Transactions on Components, Packaging and Manufacturing Technology. doi: 10.1109/TCPMT.2019.2898979

7. [9] Deffenbaugh, P.I., Rumpf, R.C., Church, K.H.: 'Broadband microwave frequency characterization of 3-D printed materials', IEEE Trans. Compon. Packag. Manuf. Technol. 2013, 3, (12), pp. 2147-2155

8. [13] Huber, E., Mirzaee, M., Bjorgaard, J., et al.: 'Dielectric property measurement of PLA'. 2016 IEEE Int. Conf. Electro Information Technology (EIT), Grand Forks, ND, 2016, pp. 0788-0792

9. [16] Zhang, S.Y., Njoku, C.C., Whittow, W.G., et al.: 'Novel 3D printed synthetic dielectric substrates', Microw. Opt. Technol. Lett., 2015, 57, (10), pp. 2344-2346

10. L. Catarinucci, R. Colella, P. Coppola, L. Tarricone, "Microwave Characterization of Polylactic Acid for 3D-Printed Dielectrically-Controlled Substrates, “ IET Microwaves, Antennas \& Propagation. 11. 10.1049/iet-map.2017.0498.

11. K. V. Hoel, S. Kristoffersen, J. Moen, K. G. Kjelgård and T. S. Lande, "Broadband antenna design using different 3D printing technologies and metallization processes," 2016 10th European Conference on Antennas and Propagation (EuCAP), Davos, 2016, pp. 1-5. doi: 10.1109/EuCAP.2016.7481620

12. Patrick F. Flowers, Christopher Reyes, Shengrong Ye, Myung Jun Kim, Benjamin J. Wiley, "3D printing electronic components and circuits with conductive thermoplastic filament", Additive Manufacturing, vol. 18, pp. 156-163, 2017.

13. S. Roy, M. B. Qureshi, S. Asif and B. D. Braaten, "A model for 3D-printed microstrip transmission lines using conductive electrifi filament," 2017 IEEE International Symposium on Antennas and Propagation \& USNC/URSI National Radio Science Meeting, San Diego, CA, pp. 1099-1100, 2017.

14. BlackMagic Graphene enhanced PLA Datasheet [Online]. Available: http://www.blackmagic3d.com/Conductive-p/grphn-pla.htm. Last accessed: March 2019

15. CST Microwave Studio [Online]. Available: https://www.cst.com. Last accessed: March 2019

16. L. Catarinucci, R. Colella, P. Coppola and L. Tarricone, "Microwave characterisation of polylactic acid for 3D-printed dielectrically controlled substrates," in IET Microwaves, Antennas \& Propagation, vol. 11, no. 14, pp. 1970-1976, 19112017.

17. Silver Conductive Epoxy Adhesive 8331 [Online]. Available: https://www.mgchemicals.com. Last accessed: March 2019 
(1) Title of the paper: Electromagnetic Analysis and Performance Comparison of Fully 3D-Printed Antennas

(2) Authors: Riccardo Colella, Innovation Engineering Department, University of Salento - Lecce, Italy, riccardo.colella@unisalento.it;

Francesco Chietera, Innovation Engineering Department, University of Salento - Lecce, Italy, f.chietera@gmail.com;

Andrea Casula, Department of Electrical and Electronic Engineering, University of Cagliari, Cagliari, Italy, a.casula@diee.unica.it;

Andrea Michel, Department of Information Engineering, University of Pisa, Pisa, Italy, andrea.michel@iet.unipi.it

Giorgio Montisci, Department of Electrical and Electronic Engineering, University of Cagliari, Cagliari, Italy, giorgio.montisci@unica.it;

Gianluca Gatto, Department of Electrical and Electronic Engineering, University of Cagliari, Cagliari, Italy, gatto@diee.unica.it;

Paolo Nepa, Department of Information Engineering, University of Pisa, Pisa, Italy, p.nepa@iet.unipi.it; Hendrik Rogier, Internet Technology and Data Science Lab, Ghent University, Gent, Belgium, hendrik.rogier@ugent.be

Luca Catarinucci, Innovation Engineering Department, University of Salento - Lecce, Italy, luca.catarinucci@unisalento.it;

(3) Corresponding and Presenting author: Riccardo Colella, Innovation Engineering Department, University of Salento - Lecce, Italy, riccardo.colella@unisalento.it;

(4) Poster presentation. 